\title{
Draft Genome Analysis of Antimicrobial Streptomyces Isolated from Himalayan Lichen ${ }^{\mathrm{S}}$
}

\author{
Byeollee Kim ${ }^{1}$, So-Ra Han ${ }^{1}$, Janardan Lamichhane ${ }^{2}$, Hyun Park ${ }^{3}$, and Tae-Jin Oh ${ }^{1,4,5 *}$ \\ ${ }^{1}$ Department of Life Science and Biochemical Engineering, SunMoon University, Asan 31460, Republic of Korea \\ ${ }^{2}$ Department of Biotechnology, Kathmandu University, Kathmandu, Nepal \\ ${ }^{3}$ Unit of Polar Genomics, Korea Polar Research Institute, Incheon 21990, Republic of Korea \\ ${ }^{4}$ Genome-based BioIT Convergence Institute, Asan, Republic of Korea \\ ${ }^{5}$ Department of Pharmaceutical Engineering and Biotechnology, SunMoon University, Asan 31460, Republic of Korea
}

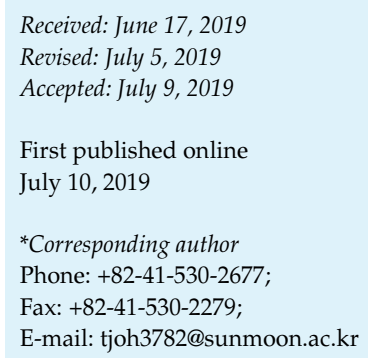

S upplementary data for this paper are available on-line only at http://jmb.or.kr.

pISSN 1017-7825, eISSN 1738-8872

Copyright (C) 2019 by

The Korean Society for Microbiology and Biotechnology
There have been several studies regarding lichen-associated bacteria obtained from diverse environments. Our screening process identified 49 bacterial species in two lichens from the Himalayas: 17 species of Actinobacteria, 19 species of Firmicutes, and 13 species of Proteobacteria. We discovered five types of strong antimicrobial agent-producing bacteria. Although some strains exhibited weak antimicrobial activity, NP088, NP131, NP132, NP134, and NP160 exhibited strong antimicrobial activity against all multidrug-resistant strains. Polyketide synthase (PKS) fingerprinting revealed results for 69 of 148 strains; these had similar genes, such as fatty acid-related PKS, adenylation domain genes, PfaA, and PksD. Although the association between antimicrobial activity and the PKS fingerprinting results is poorly resolved, NP160 had six types of PKS fingerprinting genes, as well as strong antimicrobial activity. Therefore, we sequenced the draft genome of strain NP160, and predicted its secondary metabolism using antiSMASH version 4.2. NP160 had 46 clusters and was predicted to produce similar secondary metabolites with similarities of $5-100 \%$. Although NP160 had 100\% similarity with the alkylresorcinol biosynthetic gene cluster, our results showed low similarity with existing members of this biosynthetic gene cluster, and most have not yet been revealed. In conclusion, we expect that lichen-associated bacteria from the Himalayas can produce new secondary metabolites, and we found several secondary metabolite-related biosynthetic gene clusters to support this hypothesis.

Keywords: Antimicrobial activity, draft genome sequencing, fingerprinting, Himalayan lichenassociated bacteria, polyketide synthase, secondary metabolites

\section{Introduction}

Approximately 13,500 species of lichen are estimated to inhabit $8 \%$ of the earth's surface in various environments, including low temperature, drought, and darkness [1]. To survive in such environments, lichens produce unique secondary metabolite-related compounds that have antimicrobial, antitumoral, immunostimulating, and/or antiviral activities. In addition, they contribute to symbiosis of the mycobiont (fungal partner), photobiont (photosynthetic partner, usually a green algae or cyanobacterium), and non-photosynthetic partner (bacterium). Before 2005, research regarding lichens was focused on lichenicolous fungus [2] and cyanobacteria [3]; the word 'lichenassociated bacteria' began to appear in the literature, due to the work by Alexandra et al., beginning in 2011 [4]. It has recently been reported that millions of lichen-associated bacteria greatly influence the survival of lichen through various roles such as resistance stress factor, detoxification of metabolites, support for photosynthesis, and nutrient supply [5]. In 2018, Delphine et al. reported using genome analysis to identify a new metabolite from the alpha- 
proteobacterium strain MOLA1416, a marine type of lichen-associated Proteobacteria [6]. Recent studies of lichenassociated bacteria have been performed to confirm activity and select cultivable bacteria for in-depth genome analysis.

Most research studies regarding lichen-associated bacteria are based on the hypothesis that such bacteria will produce one or more unusual secondary metabolites in the symbiotic relationship. The biosynthesis of secondary metabolites, analyzed by biosynthetic gene cluster, is already well known; this includes multidomain enzymes such as polyketide synthase (PKS) and non-ribosomal peptide synthetase (NRPS) [7-10]. Although PKS and NRPS comprise most of the metabolite class, knowledge regarding the biological activities of PKS and NRPS genes remains insufficient. Lichen-associated bacteria have been analyzed from diverse environments, including thermal spots [5], sub-Arctic [11], and Arctic [12]. Thus far, 465 species of lichen have been identified in Nepal [13]; nevertheless, there has been no study of the diversity of lichenassociated bacteria or their PKS and NRPS genes. Notably, lichen flora, market potential, and various activities (antioxidant, antimicrobial, and toxicity) were reported regarding lichens $[14,15]$. Other lichen-associated bacteria have been reported as examples of bacterial diversity [16], as sources of novel metabolites [6], and as samples for genome analysis [17].

We undertook an extensive study regarding the antimicrobial potential of lichen-associated bacteria. We aimed to identify lichen-associated bacteria from Himalayan lichens and to analyze the genomes of selected bacteria that showed antimicrobial activities with secondary metaboliterelated biosynthetic gene clusters. Based on identification of PKS and NRPS genes using fingerprinting polymerase chain reaction (PCR), we propose the use of lichenassociated bacteria to produce antimicrobial agents. This study also includes draft genome analysis of lichenassociated bacteria to understand the biosynthesis of secondary metabolites.

\section{Materials and Methods}

\section{Isolation of Lichen-Associated Bacteria}

Two lichens from the Himalayas (Fig. S1 and Table S1) were collected $(27.718805,85.322705)$, categorized, and identified by morphological analysis. To isolate associated bacteria from lichens, a modified version of the method of Kim et al. [18] was used. The thallus segments of the lichens were spread with sterilized scissors, and $2.0 \mathrm{ml}$ of $0.85 \% \mathrm{NaCl}$ wash solution was added. The lichens were vortexed for $1 \mathrm{~min}$ and the supernatant was discarded; this step was repeated twice. Then, $100-\mu l$ aliquots of the washed, broken lichen solution were spread on replicate plates containing MY media (3.0 g malt extract, 3.0 g yeast extract, $10.0 \mathrm{~g}$ peptone, $10.0 \mathrm{~g}$ dextrose, and $20.0 \mathrm{~g}$ agar, $\mathrm{pH} 7.2), \mathrm{R} 2 \mathrm{~A}$ media ( $0.5 \mathrm{~g}$ proteose peptone, $0.5 \mathrm{~g}$ casamino acid, $0.5 \mathrm{~g}$ yeast extract, $0.5 \mathrm{~g}$ dextrose, $0.5 \mathrm{~g}$ soluble starch, $0.3 \mathrm{~g}$ sodium pyruvate, $0.3 \mathrm{~g}$ dipotassium phosphate, and $0.05 \mathrm{~g}$ magnesium sulfate, $\mathrm{pH}$ 7.2), or Bennett's media (10.0 g glucose, 1.0 g yeast extract, $2.0 \mathrm{~g}$ Bacto-peptone, and $1.0 \mathrm{~g}$ beef extract, $\mathrm{pH} 7.2)$; the plates were incubated at different temperatures $\left(8,15,25\right.$, and $\left.37^{\circ} \mathrm{C}\right)$ for 3 to 30 days.

\section{$16 S$ rRNA Gene Sequence and Phylogenetic Analysis}

All isolated bacteria were identified by $16 \mathrm{~S}$ rRNA gene sequence analysis. For $16 \mathrm{~S}$ rRNA analysis, the genomic DNA of each strain was extracted from a 100- $\mu$ l culture sample from a 1530 day pure culture of a single colony and centrifuged for $10 \mathrm{~min}$ at 10,000 $\times g$; extraction was performed using the DNeasy Blood and Tissue Kit (Qiagen, Inc., USA). The 16S rRNA was amplified with two universal primers: 518F (5'-CCAGCAGCCGCGGTA ATACG-3') and 800R (5'-TACCAGGGTATCTAATCC-3'). For difficult to analyze sequences, other primers were used: 27F (5'AGAGTTTGATCMTGGCTCAG-3') and 1492R (5'-TACGGYTAC CTTGTTACGACTT- ${ }^{\prime}$ ). Sequencing was performed by Genotech Ltd. (Korea); the results were compared with the GenBank database using BLAST to identify the most similar sequences. Closest relative strains were also identified using EzBioCloud (ChunLab, Inc., Korea), and the species of the isolates were predicted. The sequences were pairwise aligned, and phylogenetic analysis was performed using a modified version of the method described by Kimura et al. [19]. A phylogenetic tree was inferred using the neighbor-joining method in MEGA X [20].

\section{Extraction of Isolated Strains and Disk Diffusion Assay}

For the antimicrobial assay, each of the 49 lichen-associated bacteria isolates was cultured in $200 \mathrm{ml}$ liquid medium in conditions identical to those in which it was isolated. A double volume of analytical-grade ethyl acetate (Daejung, Korea) was added into the grown culture fluid, and shaken using a funnel at room temperature for $2 \mathrm{~h}$ for extraction. The solvent layer was concentrated using a rotary evaporator (EYELA A-1000S, Tokyo Rikakikai Co., Japan), and each extract was dissolved in $2.0 \mathrm{ml}$ ethyl acetate. The antimicrobial activities of all extracts obtained from the 49 isolated strains were investigated using 16 multidrugresistant microorganisms, including anaerobic and aerobic bacteria, by the disc diffusion method. Anaerobic bacteria were Staphylococcus aureus (KCTC1927), Streptococcus mutans (KCTC3065), Streptococcus sanguinis (KCTC3284), Streptococcus sobrinus (KCTC3308), Streptococcus cricetid (KCTC3640), Streptococcus ratti (КСТС3655), Aggregatibacter actinomycetemconitans (KCTC3698), Streptococcus anginosus (KCTC3983), Actinomyces viscosus (KCTC5531), and Actinomyces israelii (KCTC9054); aerobic bacteria were Bacillus subtilis (KCTC1918), Staphylococcus aureus (KCTC1928), Micrococcus luteus (KCTC1915), Escherichia coli (KCTC2441), Pseudomonas aeruginosa 
(KCTC1637), and Enterobacter cloacae (KCTC1685). The bacteria were purchased from the Korea Collection for Type Culture and Korea Research Institute of Bioscience and Biotechnology (Korea). All microorganisms were grown in Luria-Bertani (MBcell Ltd., Korea) or tryptic soy (Becton Dickinson Co., USA) or brain-heart infusion broth (Becton Dickinson Co.) under the appropriate conditions. The paper disk diffusion test was performed in accordance with the method of Bauer et al., with some modifications [21]. The growth of each of the 16 multidrugresistant microorganisms was standardized to $0.5 \mathrm{McF}$ arland and spread with a swab. Each extract was added to a paper disk (6-mm diameter, Advantec Co., Japan) and the disks were transferred onto plates inoculated with the multidrug-resistant bacterial strains. Disks containing ethyl acetate were used as negative controls. All inoculated culture plates were incubated at $37^{\circ} \mathrm{C}$, and the inhibition zones of bacterial growth were measured approximately $9 \mathrm{~h}$ later. All analyses were performed in triplicate, and the experimental values are reported as means \pm standard deviation.

\section{Fingerprinting of PKS and NRPS Genes}

In the fingerprinting experiment, eight sets of degenerate primers (Table 1) targeting genes encoding the ketoacyl synthase (KS) domains of type I PKS and type II PKS (i.e., PKS-1 and PSK-2) and the adenylation domain of NRPS were used to screen the biosynthetic potentials of the isolates [9, 10, 21-24]. PCR was performed as follows: the $20 \mu \mathrm{l}$ reaction sample contained $10 \mu \mathrm{l}$ Noble premix (Noble Bio Inc., Korea), $4 \mu$ l total DNA, $4 \mu$ l distilled water, and $1 \mu \mathrm{l}$ of each primer. Amplification comprised denaturation at $95^{\circ} \mathrm{C}$ for $1 \mathrm{~min}$, annealing at $50-68^{\circ} \mathrm{C}$ for $1 \mathrm{~min}$, and extension at $72^{\circ} \mathrm{C}$ for $2 \mathrm{~min}$. The sizes of the PCR products were $600-1,000 \mathrm{bp}$ for PKS and NRPS genes; these were purified by electrophoresis in a $1 \%(\mathrm{wt} / \mathrm{vol})$ agarose gel. Purified PCR products were ligated into pMD20-T vectors (Takara Inc., Japan), and transformed into competent E. coli XLI-blue. Positive recombinants were screened on 5-bromo-4-chloro-3-indoly- $\beta$-D-galactopyranoside (X-Gal), isopropyl- $\beta$-D-thiogalactopyranoside, and ampicillin indicator plates by color-based recombinant selection. Plasmid purification was performed using an isolation kit (GeneAll Co., Korea) and sequence analysis was carried out by Genotech Ltd. The BLASTX algorithm with default parameters was used to identify database entries related to the isolated sequences. A phylogenetic tree was generated based on the PKS and NRPS fingerprinting results; it was constructed using multiple sequence alignment tools, the Mega package, and the neighbor-joining method.

\section{Draft Genome Sequencing of NP160}

We selected lichen-associated bacteria with the highest probability to be used as a new antimicrobial agent. Genomic DNA was extracted from Streptomyces sp. NP160 using a QIAamp DNA Mini Kit (Qiagen, Inc.), and the quantity and purity were determined using an Agilent 2100 Bioanalyzer (Agilent Technologies, USA) [25]. Sequencing was performed using an Illumina MiSeq system with a $300 \mathrm{bp}$ paired-end library. De novo assembly of genome sequences was performed using Celera Assembler software (Ver. 8.3) with Illumina short reads. Prior to assembly,

Table 1. Primers used for fingerprinting of PKS and NRPS genes.

\begin{tabular}{|c|c|c|c|c|c|c|}
\hline Type & $\begin{array}{c}\text { Primer } \\
\text { name }\end{array}$ & Target gene & & $\begin{array}{l}\text { Size } \\
\text { (bp) }\end{array}$ & $\begin{array}{l}\text { Annealing } \\
\left({ }^{\circ} \mathrm{C}\right)^{*}\end{array}$ & Reference \\
\hline \multirow{7}{*}{$\begin{array}{l}\text { Type I } \\
\text { PKS }\end{array}$} & $\mathrm{K} 1 \mathrm{~F}$ & PKS-I ketoacyl synthase (KS) domains & TSAAGTCSAACATCGGBCA & & 65 & {$[10]$} \\
\hline & PKS-I-A & KS domains & GCSATGGAYCCSCARCARCGSVT & 700 & 60 & {$[21]$} \\
\hline & PKS-I-B & & GTSCCSGTSCCRTGSSCYTCSAC & & & \\
\hline & KSMAF & Beta-KS domain & TSGCSATGGACCCSCAGCAG & $\sim 700$ & 68 & [22] \\
\hline & KSMBR & & CCSGTSCCGTGSGCCTCSAC & & & \\
\hline & KSI1f & Beta-ketosynthase domains & GCI ATGGAYCCICARCARMGIVT & 700 & 50 & [9] \\
\hline & KSI2r & & GTICCIGTICCRTGISCYTCIAC & & & \\
\hline \multirow{3}{*}{$\begin{array}{l}\text { Type II } \\
\text { PKS }\end{array}$} & $540 \mathrm{~F}$ & Partial KS genes of Type II PKS & GGITGCACSTCIGGIM TSGAC & & 68 & [23] \\
\hline & 1100R & & CCGATSGCICCSAGIGAGTG & & & \\
\hline & $\mathrm{KS} \beta$ & to target conserved sequences & TACSAGTCSWTCGCCTGGTTC & & & \\
\hline \multirow[t]{4}{*}{ NRPS } & $\mathrm{A} 3 \mathrm{~F}$ & NRPS adenylation domains/alignments & GCSTACSYSATSTACACSTCSGG & & 65 & {$[24]$} \\
\hline & A7R & $\begin{array}{l}\text { of ketosynthase, acyltransferase and } \\
\text { adenylation sequences }\end{array}$ & SASGTCVCCSGTSCGGTAS & & & \\
\hline & MTF2 & Adenylation A domain of NRPS & GCNGGYGGYGCNTAYGTNCC & 1,000 & 60 & {$[24]$} \\
\hline & MTR & & CCNCGDATYTTNACYTG & & & \\
\hline
\end{tabular}

*Annealing temperature was modified based on the results of preliminary experiments for this study. 
Illumina reads were trimmed using FASTX-Toolkit software (http://hannonlab.cshl.edu/fastx_toolkit) with parameters -t 20, -I 70, and -Q 33; a paired sequence was then selected from trimmed Illumina reads. Coding DNA sequences (CDSs) were predicted and annotated using the National Center for Biotechnology Information (NCBI) Prokaryotic Genome Annotation Pipeline and the Rapid Annotation using the Sub-system Technology server [26]. The numbers and types of secondary metabolites present in microbial genomes were determined using antiSMASH software (version 4.2) [27]. For the analysis of antiSMASH results, each gene was predicted using BLASTX and compared with known gene clusters.

\section{Results and Discussion}

\section{Identification of Lichen-Associated Bacteria and Phylogenetic Analysis}

We isolated 185 lichen-associated bacteria from the rarely studied Himalayan region. Based on their color and morphology when grown on media, we selected 49 isolates for further analysis (Table S2). The isolated bacteria ratios were balanced (Fig. 1), which contrasts with the findings of prior studies in which most such bacteria were classified as Alphaproteobacteria [28]. Forty-nine of the isolates underwent phylogenetic analysis. Based on BLAST analysis of $16 \mathrm{~S}$ rRNA gene sequences, we found that the isolates were divided into three dominant groups: 17 species of Actinobacteria, 19 species of Firmicutes, and 13 species of Proteobacteria (Fig. 1A). The 17 species of Actinobacteria were classified as Curtobacterium spp., Leifsonia spp., Streptomyces spp., and Rhodococcus spp. Furthermore, a phylogenetic tree constructed using the neighbor-joining method enabled us to identify isolates at the genus level with over $98 \%$ identity (Fig. 1B). According to Suzuki et al., there have been some studies of lichen-associated bacteria from various lichens, and secondary metabolites of Amycolatopsis have been reported [29].

\section{Antimicrobial Properties of Lichen-Associated Bacteria}

To evaluate the antimicrobial potentials of the isolated bacteria, a paper disk diffusion test was performed. In this test, our extracts showed similar antimicrobial strengths, with zone of inhibition diameters of 6-20 mm against both anaerobic and aerobic bacteria. As shown in Table 2, most strains had no antimicrobial activity or narrow antimicrobial activity; however, 12 isolates showed inhibitory antimicrobial activity against both Gram-negative and Gram-positive bacteria. In particular, strains NP088, NP131, NP132, NP134, and NP160 had the strongest inhibitory activity, which suggested that they produced antimicrobial agents.
These isolates were classified as Bacillus, Methylobacterium, and Streptomyces. Strain NP160 showed the most potent antimicrobial activity, with a zone of inhibition diameter of $22.89 \pm 1.07 \mathrm{~mm}$ against Streptococcus anginosus, which causes pyogenic liver abscess. In our antimicrobial activity assays, each bacterial species exhibited the strongest antimicrobial resistance known.

Lichen-associated bacteria have been isolated from a variety of lichen. Each bacterium plays a role in symbiosis and has potential for biotechnological applications; these primarily include nitrogen fixation and phosphate solubilization, which can promote plant growth [30]. Only Kim et al. reported the antimicrobial activity of lichenassociated bacteria; these organisms showed zone of inhibition diameters of 8-12 $\mathrm{mm}$ [18]. Our isolates showed stronger inhibitory antimicrobial activity and were predicted to produce antimicrobial agents. We presume that each lichen-associated bacterial species has a unique role within the lichen. Therefore, we further assessed the antimicrobial activity and corresponding biosynthetic analysis using fingerprinting of PKS and NRPS genes.

\section{Fingerprinting of PKS and NRPS Genes}

Many intriguing secondary metabolites are biosynthesized by the PKS, NRPS, and PKS-NRPS hybrid systems. To assess the biosynthetic potentials of lichen-associated bacteria regarding PKS and NRPS systems, we evaluated each of the 49 isolated strains by amplifying PKS (type I and type II) and NRPS genes with eight sets of diverse primers. The results revealed 148 PCR products. Only the products of our desired size were refined and analyzed, and the results were obtained for 69 samples. We confirmed the identities of PKS and NRPS genes by BLASTX comparison with those in the GenBank database. Most PKS genes showed high similarities of $40-100 \%$. We identified 19 acyltransferase domain genes, nine adenylation-related domain genes, five beta-ketoacyl domain genes, 13 fatty acid-related PKS genes and four hybrid PKS-NRPS genes (Table S3). PKS fingerprinting results revealed that most strains had more than one PKS-NRPS-related gene. Most fingerprinting genes were involved in bacillaene biosynthesis (e.g., PksC, PksF, PksG, PksH, PksI, PksJ, PksL, PksM, PksN, and $P k s R)$ [31]. Strain NP160 had the greatest number of PKS and NRPS genes without any bands indicative of nonspecific amplification (Fig. S2); it also demonstrated the strongest antimicrobial activity. Fingerprinting showed that NP160 had six types of PKS- and NRPS-related genes. Although the primers were constructed on the basis of PKS and NRPS domains, it was difficult to determine whether 
A

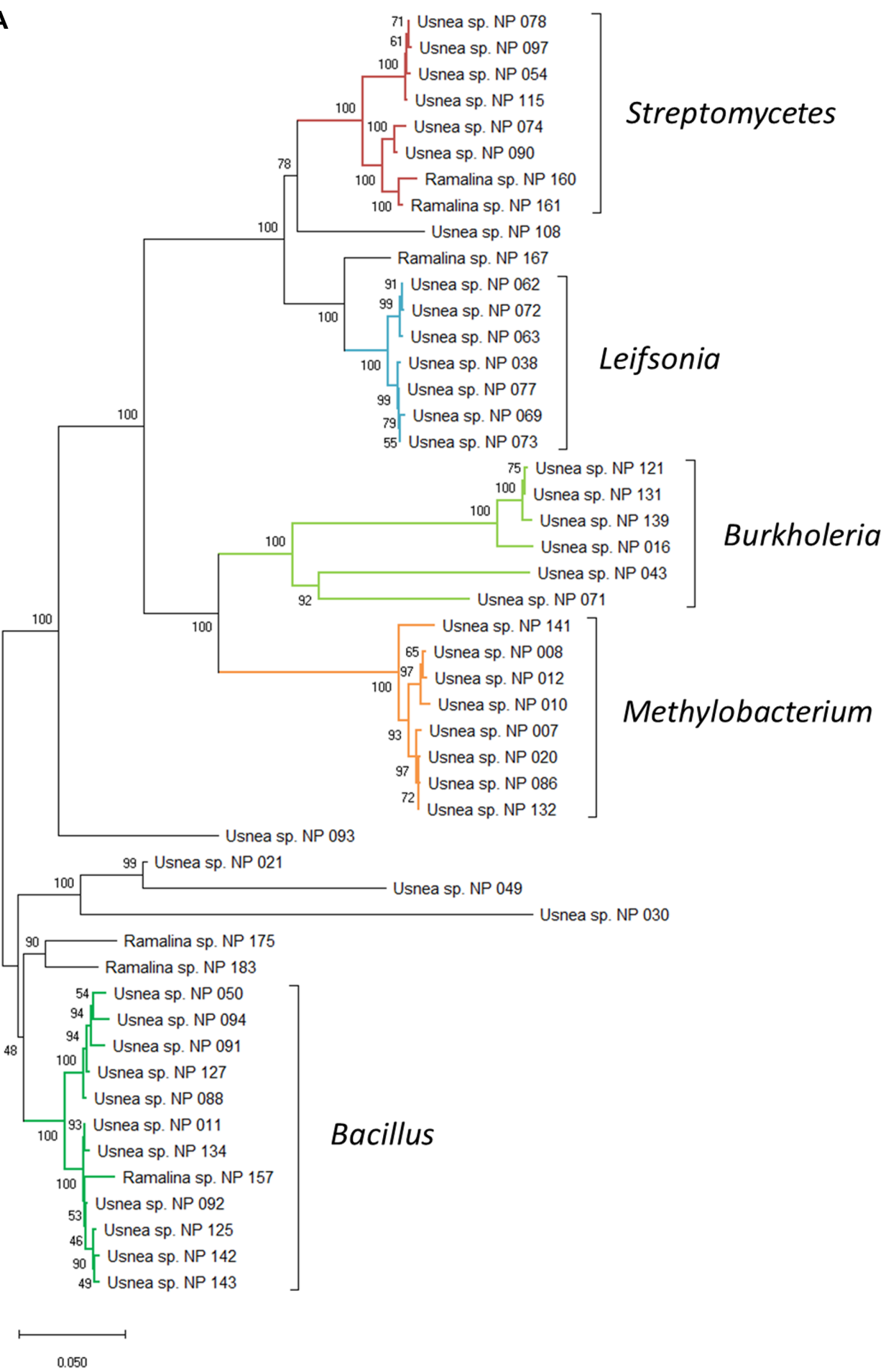

Fig. 1. Classification of lichen-associated bacteria from the Himalayas.

(A) Phylogenetic tree of lichen-associated bacteria from Usnea sp. and Ramalina sp. (B) Phylogenetic tree of Actinomycetes including BlastN results. Neighbor-joining phylogenetic tree analysis of $16 \mathrm{~S}$ rRNA of lichen-associated bacteria.

enzyme function was consistent among the isolates. Therefore, we aimed to analyze the draft genome in detail; we selected strain NP160, which was predicted to produce several secondary products. 
B

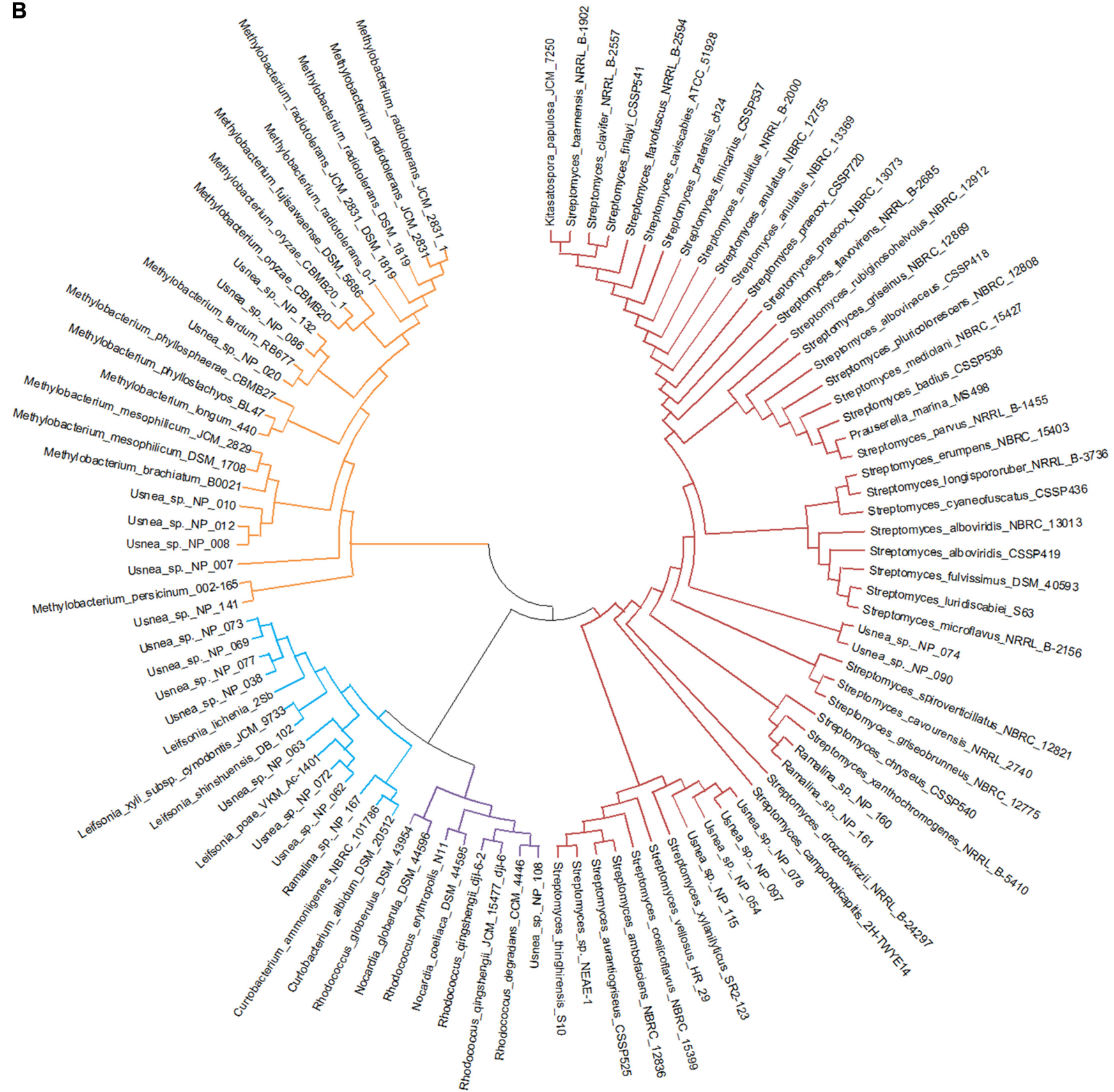

Fig. 1. Continued.

\section{Draft Genome Analysis of Streptomyces sp. NP160}

In previous studies, some lichen-associated bacteria were shown to produce secondary metabolites, and corresponding genomic analyses were performed [32]. Researchers have found much of interest in the genomes and secondary metabolites of lichen-associated bacteria. Among the 49 lichen-associated bacteria, we found many isolates that could produce various substances. Although many researchers have reported on lichen-associated bacteria, this study describes secondary metabolite substanceproducing strains and fatty acid synthesis-related PKS genes. Strain NP160 had robust expression of six types of PKS and NRPS genes, as well as strong antimicrobial activity. PKS fingerprinting methodology cannot readily be 
Table 2. Antimicrobial activity per bacterial genus.

\begin{tabular}{|c|c|c|c|c|c|c|c|c|c|c|c|c|c|c|c|c|}
\hline & 1 & 2 & 3 & 4 & 5 & 6 & 7 & 8 & 9 & 10 & 11 & 12 & 13 & 14 & 15 & 16 \\
\hline NP007 & - & + & - & + & + & + & + & + & + & + & + & + & - & + & + & + \\
\hline NP008 & - & + & + & + & + & + & + & + & + & + & + & + & - & + & + & + \\
\hline NP010 & - & - & - & - & - & - & - & - & - & - & - & - & - & - & - & - \\
\hline NP011 & - & - & - & - & - & - & - & - & - & - & - & - & - & - & - & - \\
\hline NP012 & + & - & - & + & + & - & - & - & - & - & - & - & - & - & - & + \\
\hline NP016 & - & + & + & + & + & + & - & - & - & - & - & - & - & - & - & - \\
\hline NP020 & - & - & - & - & - & - & - & + & - & - & - & - & - & + & - & - \\
\hline NP021 & - & - & - & - & - & - & - & - & - & - & - & - & - & - & - & - \\
\hline NP030 & - & + & + & + & + & - & - & - & - & - & - & - & - & - & - & + \\
\hline NP038 & + & - & + & + & + & + & + & + & - & - & - & + & - & - & - & - \\
\hline NP043 & + & + & + & + & + & + & + & + & - & - & + & + & + & - & + & + \\
\hline NP049 & - & - & - & - & - & - & - & - & - & - & - & - & - & - & - & - \\
\hline NP050 & - & + & - & - & + & + & - & + & + & + & - & - & - & + & - & - \\
\hline NP054 & ++ & + & + & ++ & + & + & + & + & + & ++ & + & - & + & + & + & + \\
\hline NP062 & + & - & + & + & + & + & + & + & - & - & - & + & + & + & - & + \\
\hline NP063 & - & - & + & + & - & + & + & ++ & + & - & + & + & + & + & + & + \\
\hline NP069 & - & - & + & + & - & - & - & + & - & - & - & - & - & - & + & - \\
\hline NP071 & - & + & + & + & + & - & - & + & - & - & - & - & - & - & - & - \\
\hline NP072 & + & + & + & + & - & + & + & + & + & - & + & + & + & + & + & + \\
\hline NP073 & + & + & + & + & + & + & + & - & ++ & - & + & + & + & + & + & ++ \\
\hline NP074 & + & - & + & + & - & - & + & + & - & - & - & + & - & - & - & + \\
\hline NP077 & + & + & + & + & + & + & - & - & - & - & - & + & - & - & - & - \\
\hline NP078 & - & - & + & - & - & - & - & + & - & - & - & - & - & - & - & - \\
\hline NP086 & + & - & + & + & - & - & + & + & + & + & - & - & + & - & - & - \\
\hline NP088 & +++ & +++ & - & +++ & +++ & - & +++ & +++ & +++ & +++ & +++ & +++ & +++ & - & +++ & +++ \\
\hline NP090 & - & - & - & - & - & - & - & - & - & - & - & - & - & - & - & - \\
\hline NP091 & - & - & - & - & - & - & - & - & - & - & - & - & - & - & - & - \\
\hline NP092 & +++ & - & - & ++ & + & - & - & - & - & - & - & - & - & - & - & + \\
\hline NP093 & + & + & + & + & + & + & + & + & + & - & + & + & - & + & + & + \\
\hline NP094 & - & - & - & - & - & - & - & - & - & - & - & - & - & - & - & - \\
\hline NP097 & - & - & - & - & - & - & - & - & - & - & - & - & - & - & - & - \\
\hline NP108 & - & - & - & - & - & - & - & - & - & - & - & - & - & - & - & - \\
\hline NP115 & - & - & - & - & - & - & - & - & - & - & - & - & - & - & - & - \\
\hline NP121 & - & + & + & + & + & + & - & - & - & - & - & - & - & - & - & - \\
\hline NP125 & - & - & - & - & - & - & - & - & - & - & - & - & - & - & - & - \\
\hline NP127 & - & - & - & - & + & + & - & - & - & - & - & - & - & - & + & + \\
\hline NP131 & +++ & +++ & +++ & ++ & + & + & - & - & - & - & - & - & - & - & - & - \\
\hline NP132 & +++ & ++++ & +++ & +++ & ++++ & ++++ & ++++ & ++++ & +++ & ++++ & - & ++++ & - & ++++ & - & ++++ \\
\hline NP134 & +++ & ++ & - & ++ & + & + & - & - & - & - & - & - & - & - & - & - \\
\hline NP139 & - & + & + & + & + & + & - & - & + & - & - & + & - & + & - & + \\
\hline NP141 & - & - & - & - & - & - & - & - & - & - & - & - & - & - & - & - \\
\hline NP142 & - & - & - & - & - & - & - & - & - & - & - & - & - & - & - & - \\
\hline NP143 & ++ & + & - & - & - & - & - & - & - & - & - & - & - & - & - & - \\
\hline NP157 & - & - & - & - & - & - & - & - & - & - & - & - & - & - & - & - \\
\hline
\end{tabular}


Table 2. Continued.

\begin{tabular}{cccccccccccccccccc}
\hline & 1 & 2 & 3 & 4 & 5 & 6 & 7 & 8 & 9 & 10 & 11 & 12 & 13 & 14 & 15 & 16 \\
\hline NP160 & ++ & + & + & + & + & + & ++++ & ++++ & +++ & - & - & ++++ & - & ++++ & - & ++++ \\
NP161 & - & - & - & - & - & - & - & - & - & - & - & - & - & - & - & - \\
NP167 & ++ & + & + & + & + & + & + & + & + & + & + & + & - & + & + & + \\
NP175 & - & - & - & - & - & - & - & + & - & + & - & - & - & + & - & - \\
NP183 & - & - & - & - & + & + & + & + & + & + & + & + & + & + & - & -
\end{tabular}

*1, Bacillus subtilis; 2, Staphylococcus aureus; 3, Micrococcus luteus; 4, Escherichia coli; 5, Pseudomonas aeruginosa; 6, Enterobacter cloacae; 7, Staphylococcus aureus; 8, Streptococcus mutans; 9, Streptococcus sanguinis; 10, Streptococcus sobrinus; 11, Streptococcus criceti; 12, Streptococcus ratti; 13, Aggregatibacter actinomycetemcomitans; 14, Streptococcus anginosus; 15 , Actinomyces viscosus; 16 , Actinomyces israelii.

$*_{-}$, negative;,$+>6 \mathrm{~mm} ;++,>8 \mathrm{~mm} ;+++,>10 \mathrm{~mm}$; and,$++++>20 \mathrm{~mm}$.

used to classify gene clusters of antimicrobial agents; therefore, we assembled a draft genome of strain NP160 to identify biosynthetic genes related to secondary metabolite

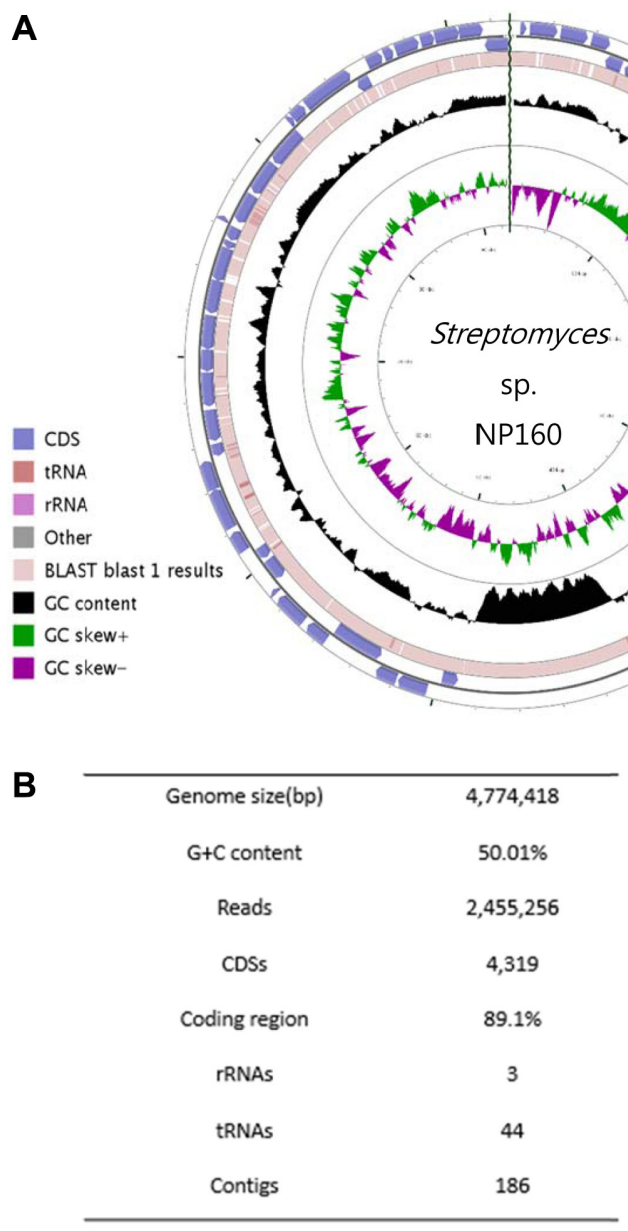

Fig. 2. Genome information of NP160.

(A) Circular representation of Streptomyces sp. NP160 draft genome. The map was created using CGview Comparison tools. (B) Characteristics of NP160 genome. gene clusters. The draft genome sequence had a size of $4,774,418$ bp with a $\mathrm{G}+\mathrm{C}$ content of $50.01 \%$, and generated a total of 2,455,256 reads. Predicted gene sequences were translated and searched against the NCBI non-redundant, Clusters of Orthologous Groups, and Kyoto Encyclopedia of Genes and Genomes databases. A total of 4,319 CDSs were predicted; the coding region comprised $89.1 \%$ of the genome. In addition, 3 rRNAs and 44 tRNAs were predicted, and genome assembly resulted in 186 contigs, as shown in Fig. 2. Based on the draft genome results, strain NP160 was classified as Streptomyces mauvecolor. Thus far, this species has only one strain; the anti-fungal activity of S. mauvecolor BU16 was previously described [33]. Importantly, our analysis demonstrated biosynthesis of secondary metabolites in a unique bacterial strain, $S$. sp. NP160.

\section{Predicted Biosynthetic Gene Cluster for Secondary Metabolites in S. sp. NP160}

S. sp. NP160 was assessed for the presence of secondary metabolite gene clusters using antiSMASH, based on draft genome information. The results showed that $S$. sp. NP160 had 46 secondary metabolite gene clusters; 19 of these gene clusters were similar to previously described secondary metabolite biosynthetic gene clusters, including sioxanthin, meiligmycin, lomaiviticin, divergolide, lasalocid, incedinine, GE81112, 5'-hydroxystreptomycin, furaquinocin, ravidomycin, calicheamicin, paromomycin, surfactin, teicoplanin, chlortetracycline, chlorizidine A, and alkylresorcinol. These gene clusters demonstrated diverse similarities of 5$100 \%$, as shown in Table 3. There were several types of clusters, such as terpene, saccharide, fatty acid, and PKS type III; these contained PKS-related clusters (e.g., divergolide, lasalocid, and meridamycin), but showed low similarity with well-known gene clusters. Therefore, we chose to predict four types of clusters that putatively 
Table 3. Summary of NP160 antiSMASH results (v 4.2).

\begin{tabular}{|c|c|c|c|c|c|}
\hline Cluster & Type & From & To & Most similar known cluster (similarity, \%) & MIBiG BGC-ID \\
\hline Cluster 1 & Terpene & 7223 & 28248 & Sioxanthin biosynthetic gene cluster (60) & BGC0001087_c4 \\
\hline Cluster 2 & Cf_putative & 45854 & 67462 & Meilingmycin biosynthetic gene cluster (2) & BGC0000093_c1 \\
\hline Cluster 3 & Cf_putative & 107150 & 117184 & Lomaiviticin biosynthetic gene cluster (3) & BGC0000241_c1 \\
\hline Cluster 4 & Cf_putative & 99211 & 113801 & Divergolide biosynthetic gene cluster (6) & BGC0001119_c1 \\
\hline Cluster 5 & Cf_putative & 116246 & 137055 & Lasalocid biosynthetic gene cluster (3) & BGC0000087_c1 \\
\hline Cluster 6 & Cf_putative & 163699 & 170292 & Incednine biosynthetic gene cluster (2) & BGC0000078_c1 \\
\hline Cluster 7 & Cf_putative & 2860 & 15687 & GE81112 biosynthetic gene cluster (7) & BGC0000360_c1 \\
\hline Cluster 8 & Cf_saccharide & 304 & 48382 & 5'-Hydroxystreptomycin biosynthetic gene cluster (13) & BGC0000690_c1 \\
\hline Cluster 9 & Cf_fatty_acid-Cf_saccharide & 70760 & 102725 & Furaquinocin A biosynthetic gene cluster (8) & BGC0001078_c1 \\
\hline Cluster 10 & Cf_putative & 24494 & 35734 & Meilingmycin biosynthetic gene cluster (2) & BGC0000093_c1 \\
\hline Cluster 11 & Cf_saccharide & 81255 & 107170 & Ravidomycin biosynthetic gene cluster (5) & BGC0000263_c1 \\
\hline Cluster 12 & Cf_putative & 125432 & 138595 & Meridamycin biosynthetic gene cluster (5) & BGC0001011_c1 \\
\hline Cluster 12 & Cf_saccharide & 1 & 32710 & Calicheamicin biosynthetic gene cluster (4) & BGC0000033_c1 \\
\hline Cluster 13 & Cf_putative & 31926 & 36355 & Paromomycin biosynthetic gene cluster (5) & BGC0000712_c1 \\
\hline Cluster 14 & Cf_putative & 15433 & 26486 & Meridamycin biosynthetic gene cluster (5) & BGC0001011_c1 \\
\hline Cluster 15 & Cf_putative & 110365 & 121107 & Surfactin biosynthetic gene cluster (8) & BGC0000433_c1 \\
\hline Cluster 16 & Cf_putative & 27142 & 62873 & Teicoplanin biosynthetic gene cluster (3) & BGC0000440_c1 \\
\hline Cluster 17 & Cf_saccharide & 85115 & 120159 & Chlortetracycline biosynthetic gene cluster (5) & BGC0000209_c1 \\
\hline Cluster 18 & Cf_fatty_acid & 142859 & 163881 & Chlorizidine A biosynthetic gene cluster (7) & BGC0001172_c1 \\
\hline Cluster 19 & T3pks-Cf_saccharide & 18544 & 79268 & Alkylresorcinol biosynthetic gene cluster (100) & BGC0000282_c1 \\
\hline
\end{tabular}

*cf, possible cluster.

encoded known pathways for the production of sioxanthin, furaquinocin A, chlorizidine A, and alkylresorcinol. Although most clusters displayed low levels of similarity with well-known clusters, cluster 46 of $S$. sp. NP160 had $100 \%$ similarity with the alkylresorcinol biosynthetic gene cluster (Fig. 3). Alkylresorcinol is classified as a resorcinolic lipid, which can help prevent cells from becoming cancerous. Nearly all gene clusters had PKS-related genes, such as acyl carrier protein and ketoacyl transferase; cluster 2, which had a terpene gene cluster, was an exception. Based on these results, our PKS fingerprinting data matched genome analysis data in that both showed several genes related to PKS, such as acyltransferase and acyl carrier protein (Table S3 and Fig. 3).

In conclusion, most lichens produce unique secondary metabolites and are known to contain multiple chemical constituents; these include mono-substituted phenyl rings, terpenes, fatty acids, and polysaccharides, with antitumor, antimicrobial, anti-inflammatory, antioxidant, and antithrombosis activities. In particular, lichens of Ramalina sp. have been reported to exhibit antioxidant activities due to the presence of salazinic acid and usnic acid (Table S1). Although most bioactive compounds in lichens are produced by fungi, lichen-associated bacteria also produce bioactive compounds. Streptomyces species can perform biosynthesis of secondary metabolites [34]. It was recently published that lichen-associated bacteria can produce a phthalazinone derivative [29]. Therefore, we hypothesized that our strain could produce secondary metabolites or other unique metabolites. Based on antimicrobial activity and PKS fingerprinting results, we selected a strain for draft genome analysis, and identified putative secondary metaboliterelated biosynthetic gene clusters. S. sp. NP160 had 30 unknown gene clusters that were predicted to have antimicrobial activity. The biosynthetic gene cluster results showed low similarity with those of known clusters; only two S. sp. NP160 biosynthetic gene clusters showed similarity $>60 \%$ with known clusters. We expect that new secondary metabolites can be isolated from S. sp. NP160. In addition, strain NP160 was predicted to contain meiligmycin and alkylresorcinol, which have been reported to exhibit antimicrobial activity. Therefore, we presume that NP160 can produce an alkylresorcinol derivative with antimicrobial activity. In the present study, we assessed antimicrobial activity and performed PKS fingerprinting of cultivable lichen-associated bacteria from the Himalayas to identify 


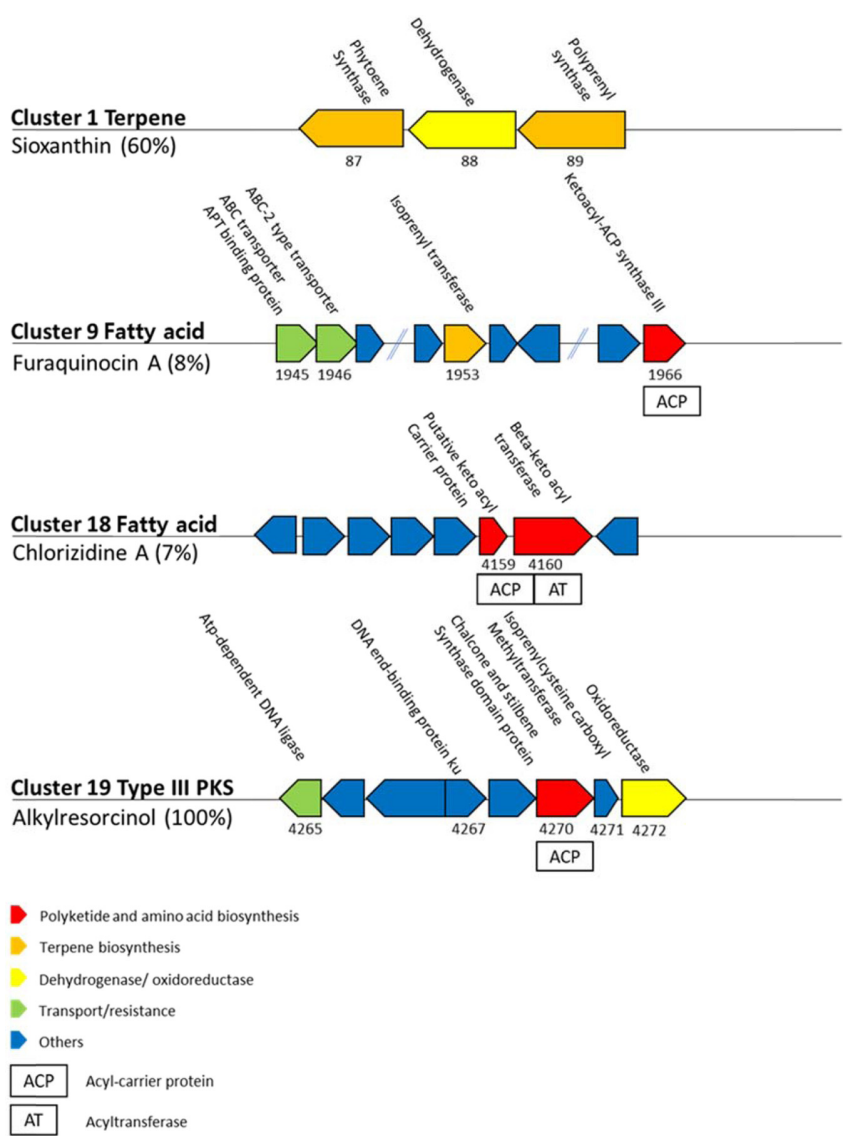

Fig. 3. Biosynthetic gene clusters predicted from Streptomyces sp. NP160.

potential new antimicrobial agents. Our results can aid in the investigation of novel antimicrobial agents and provide a basis for genome analysis of lichen-associated bacteria; moreover, the results increase the available knowledge regarding the relationship between lichens and bacteria.

\section{Nucleotide Sequence Accession Numbers}

The draft genome information of Streptomyces sp. NP160 was deposited in GenBank under the accession number VDMJ00000000.

\section{Acknowledgments}

This research was supported by a grant (NRF2016R1D1A3B03933814) from the Basic Science Research Program through the National Research Foundation of Korea funded by the Ministry of Education, Science and Technology, Republic of Korea. This work was also supported by the Korea Polar Research Institute (grant no. PE19210).

\section{Conflict of Interest}

The authors have no financial conflicts of interest to declare.

\section{References}

1. White PA, Oliveira RC, Oliveira AP, Serafini MR, Araújo AA, Gelain DP, et al. 2014. Antioxidant activity and mechanisms of action of natural compounds, isolated from lichens: a systematic review. Molecules 19: 14496-14527.

2. He H, Bigelis R, Yang HY, Chang LP, Singh MP. 2005. Lichenicolins $\mathrm{A}$ and $\mathrm{B}$, new bisnaphthopyrones from an unidentified lichenicolous fungus, strain LL-RB0668. J. Antibiot. Tokyo 58: 731-736.

3. Oksanen I, Jokela J, Fewer DP, Wahlsten M, Rikkinen J, Sivonen K. 2004. Discovery of rare and highly toxic microcystins from lichen-associated cyanobacterium Nostoc sp. strain IO-102-I. Appl. Environ. Microbiol. 70: 5756-5763.

4. Mushegian AA, Peterson CN, Baker CC, Pringle A. 2011. Bacterial diversity across individual lichens. Appl. Environ. Microbiol. 77: 4249-4252.

5. Parrot D, Legrave N, Delmail D, Grube M, Suzuki M, Tomasi S. 2016. Review - lichen-associated bacteria as a hot spot of chemodiversity: focus on uncialamycin, a promising compound for future medicinal applications. Planta Med. 82: 1143-1152.

6. Parrot D, Intertaglia L, Jehan $\mathrm{P}$, Grube M, Suzuki MT, Tomasi S. 2018. Chemical analysis of the alphaproteobacterium strain MOLA1416 associated with the marine lichen Lichina pygmaea. Phytochemistry 145: 57-67.

7. Bertrand RL, Sorensen JL. 2018. A comprehensive catalogue of polyketide synthase gene clusters in lichenizing fungi. J. Ind. Microbiol. Biotechnol. 45: 1067-1081.

8. Miyanaga A, Kudo F, Eguchi T. 2018. Protein-protein interactions in polyketide synthase-nonribosomal peptide synthetase hybrid assembly lines. Nat. Prod. Rep. 35: 11851209.

9. Zhang W, Zhang F, Li Z, Miao X, Meng Q, Zhang X. 2009. Investigation of bacteria with polyketide synthase genes and antimicrobial activity isolated from South China Sea sponges. J. Appl. Microbiol. 107: 567-575.

10. Ayuso-Sacido A, Genilloud O. 2005. New PCR primers for the screening of NRPS and PKS-I systems in actinomycetes: detection and distribution of these biosynthetic gene sequences in major taxonomic groups. Microb. Ecol. 49: 1024.

11. Sigurbjörnsdóttir MA, Vilhelmsson O. 2016. Selective isolation of potentially phosphate-mobilizing, biosurfactantproducing and biodegradative bacteria associated with a sub-Artic, terricolous lichen, Peltigera membranacea. FEMS Microbiol. Ecol. 92(6): fiw090. 
12. Sigurbjörnsdóttir

MA, Heiðmarsson

S, Jónsdóttir AR, Vilhelmsson O. 2014. Novel bacteria associated with Arctic seashore lichens have potential roles in nutrient scavenging. Can. J. Microbiol. 60: 307-317.

13. Baniya CB, Solhøy T, Gauslaa Y, Palmer MW. 2010. The elevation gradient of lichen species richness in Nepal. Lichenologist 42: 83-96.

14. Devkota S, Chaudhary RP, Werth S, Scheidegger C. 2017. Indigenous knowledge and use of lichens by the lichenophilic communities of the Nepal Himalaya. J. Ethnobiol. Ethnomed. 13: $1-10$.

15. Jha BN, Shrestha M, Pandey DP, Bhattarai T, Bhattarai HD, Paudel B. 2017. Investigation of antioxidant, antimicrobial and toxicity activities of lichens from high altitude regions of Nepal. BMC Complement Altern. Med. 17(1): 282 doi: 10.1186/s12906-017-1797-x.

16. Bates ST, Cropsey GW, Caporaso JG, Knight R, Fierer N. 2011. Bacterial communities associated with the lichen symbiosis. Appl. Environ. Microbiol. 77: 1309-1314.

17. Han SR, Yu SC, Ahn DH, Park H, Oh TJ. 2016. Complete genome sequence of Burkholderia sp. strain PAMC28687, a potential octopine-utilizing bacterium isolated from Antarctica lichen. J. Biotechnol. 226: 16-17.

18. Kim MK, Park H, Oh TJ. 2014. Antibacterial and antioxidant capacity of polar microorganisms isolated from Arctic lichen Ochrolechia sp. Pol. J. Microbiol. 63: 317-322.

19. Kimura M. 1980. A simple method for estimating evolutionary rates of base substitutions through comparative studies of nucleotide sequences. J. Mol. Evol. 16: 111-120.

20. Kumar S, Stecher G, Li M, Knyaz C, Tamura K. 2018. MEGA X: molecular evolutionary genetics analysis across computing platforms. Mol. Biol. Evol. 35: 1547-1549.

21. Bauer AW, Kirby MM, Sherris JC, Truck M. 1966. Antibiotic susceptibility testing by a standardized single disk method. Am. J. Clinic. Pathol. 45: 493-496.

22. Zhao K, Penttinen P, Guan T, Xiao J, Chen Q, Xu J, et al. 2011. The diversity and anti-microbial activity of endophytic actinomycetes isolated from medicinal plants in Panxi plateau, China. Curr. Microbiol. 62: 182-190.

23. Gaber AA, Badr OM, Emara SA, Ibrahim AM. 2015. Antitumor activity of two Streptomyces extracts (Ag18 \& Ag20) on Ehrlich ascites tumor in mice: in vitro and in vivo studies. J. Biosci. Appl. Res. 1: 20-29.
24. Wawrik B, Kerkhof L, Zylstra GJ, Kukor JJ. 2005. Identification of unique type II polyketide synthase genes in soil. Appl. Environ. Microbiol. 71: 2232-2238.

25. Han SR, Lee JH, Kang S, Park H, Oh TJ. 2016. Complete genome sequence of opine-utilizing Variovorax sp. strain PAMC28711 isolated from an Antarctic lichen. J. Biotechnol. 225: 46-47.

26. Aziz RK, Bartels D, Best AA, DeJongh M, Disz T, Edwards RA, et al. 2008. The RAST Server: rapid annotations using subsystems technology. BMC Genomics 9: 75.

27. Blin K, Wolf T, Chevrette MG, Lu X, Schwalen CJ, Kautsar SA, et al. 2017. antiSMASH 4.0-improvements in chemistry prediction and gene cluster boundary identification. Nucleic Acids Res. 45: W36-W41.

28. Ayuso A, Clark D, González I, Salazar O, Anderson A, Genilloud O. 2005. A novel actinomycete strain de-replication approach based on the diversity of polyketide synthase and nonribosomal peptide synthetase biosynthetic pathways. Appl. Microbiol. Biotechnol. 67: 795-806.

29. Zheng KX, Jiang Y, Jiang JX, Huang R, He J, Wu SH. 2019. A new phthalazinone derivative and a new isoflavonid glycoside from lichen-associated Amycolatopsis sp. Fitoterapia 135: $85-89$.

30. Sigurbjörnsdóttir MA, Andrésson ÓS, Vilhelmsson O. 2016. Nutrient scavenging activity and antagonistic factors of non-photobiont lichen-associated bacteria: a review. World J. Microbiol. Biotechnol. 32: 68.

31. Butcher RA, Schroeder FC, Fischbach MA, Straight PD, Kolter R, Walsh CT, Clardy J. 2007. The identification of bacillaene, the product of the PksX megacomplex in Bacillus subtilis. Proc. Natl. Acad. Sci. USA 104: 1506-1509.

32. Schneider O, Simic N, Aachmann FL, Rückert C, Kristiansen KA, Kalinowski J, et al. 2018. Genome mining of Streptomyces sp. YIM 130001 isolated from lichen affords new thiopeptide antibiotic. Front Microbiol. 9: 3139.

33. Jeon BJ, Kim JD, Han JW, Kim BS. 2016. Antifungal activity of rimocidin and a new rimocidin derivative BU16 produced by Streptomyces mauvecolor BU16 and their effects on pepper anthracnose. J. Appl. Microbiol. 120: 1219-1228.

34. Calcott MJ, Ackerley DF, Knight A, Keyzers RA, Owen JG. 2018. Secondary metabolism in the lichen symbiosis. Chem. Soc. Rev. 47: 1730-1760. 